\title{
New Preconditioners with Two Variable Relaxation Parameters for the Discretized Time-Harmonic Maxwell Equations in Mixed Form
}

\author{
Yuping Zeng ${ }^{1,2}$ and Chenliang $\mathbf{L i}^{2}$ \\ ${ }^{1}$ Jiangsu Key Laboratory for NSLSCS, School of Mathematical Sciences, Nanjing Normal University, \\ Jiangsu, Nanjing 210046, China \\ ${ }^{2}$ School of Mathematics and Computing Science, Guilin University of Electronic Technology, Guangxi, \\ Guilin 541004, China
}

Correspondence should be addressed to Chenliang Li, chenliang_li@hotmail.com

Received 4 May 2012; Revised 3 July 2012; Accepted 4 July 2012

Academic Editor: Ion Zaballa

Copyright (C) 2012 Y. Zeng and C. Li. This is an open access article distributed under the Creative Commons Attribution License, which permits unrestricted use, distribution, and reproduction in any medium, provided the original work is properly cited.

We provide new preconditioners with two variable relaxation parameters for the saddle point linear systems arising from finite element discretization of time-harmonic Maxwell equations in mixed form. The new preconditioners are of block-triangular forms and Schur complement-free. They are extensions of the results in Cheng et al., 2009, Grief and Schötzau, 2007, and Huang et al., 2009. Theoretical analysis shows that all eigenvalues of the preconditioned matrices are tightly clustered, and numerical tests confirm our analysis.

\section{Introduction}

We consider the preconditioning techniques for solving the saddle point linear systems arising from finite element discretization of the following time-harmonic Maxwell equations in mixed form [1-5]: find the vector field $u$ and the Lagrangian multiplier $p$ such that

$$
\begin{gathered}
\nabla \times \nabla \times u-k^{2} u+\nabla p=f \quad \text { in } \Omega, \\
\nabla \cdot u=0 \quad \text { in } \Omega, \\
u \times \vec{n}=0 \quad \text { on } \partial \Omega, \\
p=0 \quad \text { on } \partial \Omega .
\end{gathered}
$$

Here, $\Omega \subset \mathbb{R}^{2}$ is a simply connected polyhedron domain with a connected boundary $\partial \Omega$, and $\vec{n}$ denotes the outward unit normal on $\partial \Omega$. The datum $f$ is a given source (not necessarily 
divergence-free), and the wave number $k^{2}=\omega^{2} \epsilon \mu$, where $\omega \geq 0$ is the frequency, and $\epsilon$ and $\mu$ are positive permittivity and permeability parameters, respectively.

In recent years, there have been many techniques for solving Maxwell equations, such as the geometry multigrid methods [6-8], algebraic multigrid methods [9], domain decomposition methods [4, 10-13], Nodal auxiliary space preconditioning methods [14], and the solution methods to the corresponding saddle-point linear systems $[2,3,15]$. We can also use Uzawa-type iterative methods [16, 17] and preconditioned Krylov subspace methods [18-24] to solve the saddle-point linear systems. Based on the previous works in $[2,3,15]$, we will further study solution methods for the saddle-point linear systems in this paper.

Using Nédélec elements of the first kind [25-27] for the approximation of the vector field and standard nodal elements for the Lagrangian multiplier yields the following saddlepoint linear system:

$$
\mathcal{A} x \equiv\left(\begin{array}{cc}
A-k^{2} M & B^{T} \\
B & 0
\end{array}\right)\left(\begin{array}{l}
u \\
p
\end{array}\right)=\left(\begin{array}{l}
g \\
0
\end{array}\right) \equiv b
$$

where $u \in \mathbf{R}^{n}$ and $p \in \mathbf{R}^{m}$ are finite arrays, and $g \in \mathbf{R}^{n}$ is a load vector associated with $f$. The matrix $A \in \mathbf{R}^{n \times n}$ is symmetric positive semidefinite with nullity $m$ and corresponds to the curl-curl operator; $B \in \mathbf{R}^{m \times n}$ is a discrete divergence operator with full-row rank, and $M \in \mathbf{R}^{n \times n}$ is a vector mass matrix.

For convenience, we denote the standard Euclidean inner product of vectors by $\langle\cdot, \cdot\rangle$ and the null space of a matrix by null (.). For a given positive (semi)definite matrix $W$ and a vector $x$, we define the (semi)norm:

$$
|x|_{W}=\sqrt{\langle W x, x\rangle} .
$$

The matrices $A$ and $B$ have the following stability properties [3]. Let $\langle A u, u\rangle=|u|_{A}^{2}$. Then there exists an $\alpha, 0<\alpha<1$, such that

$$
|u|_{A}^{2} \geq \bar{\alpha}|u|_{M}^{2}, \quad u \in \operatorname{null}(B),
$$

where $\bar{\alpha}=\alpha /(1-\alpha)$. Matrix $B$ satisfies the discrete inf-sup condition:

$$
\inf _{0 \neq q \in \mathbf{R}^{m}} \sup _{0 \neq v \in \operatorname{null}(A)} \frac{\langle B v, q\rangle}{|v|_{M}|q|_{L}} \geq \beta>0,
$$

where the inf-sup constant $\beta>0$ is only dependent on the domain $\Omega$.

If the wave number $k^{2}>0$, then the $(1,1)$ block of $(1.2)$ is indefinite. For difficulty and corresponding solution methods of this problem, we refer to [18, 28]. Recently, by using the spectral equivalent properties similar to [4], Grief and Schötzau [3] construct the blockdiagonal preconditioner:

$$
\mathcal{M}_{k}=\left(\begin{array}{cc}
A-k^{2} M+B^{T} L^{-1} B & 0 \\
0 & L
\end{array}\right)
$$

where $L \in \mathbf{R}^{m \times m}$ is the discrete Laplace operator introduced in [3], $k^{2}<1$, and $\boldsymbol{M}_{k}$ is a symmetric positive definite block-diagonal matrix. As $L$ is augmentation-free and Schur 
complement-free, this approach overcomes the difficulty in forming the Schur complement in general. However, the computational work of $B^{T} L^{-1} B$ may be too large. Using the fact that the matrices $A+B^{T} L^{-1} B$ and $A+M$ are spectrally equivalent, [3] considers the following preconditioner:

$$
\widehat{\mathcal{M}}_{k}=\left(\begin{array}{cc}
A+\left(1-k^{2}\right) M & 0 \\
0 & L
\end{array}\right)
$$

and shows that the eigenvalues of the preconditioned matrix are tightly clustered.

Based on the work of Grief and Schötzau [3], [2] gives block-triangular Schur complement-free preconditioners for the linear system (1.2). And it is shown that all eigenvalues of the proposed block-triangular preconditioning matrices are more tightly clustered. Compared with the restriction $k^{2}<1$ in [3], [2] considers the general case $k^{2} \in \mathbf{R}^{+}$. Furthermore, [15] provides block-triangular preconditioners when $k^{2}=0$ with two variable relaxation parameters.

Based on the previous work $[2,3,15]$, mentioned above, in this paper we are devoted to give new preconditioners with two scaling parameters. The new block triangular preconditioners in the general case $k^{2} \in \mathbf{R}^{+}$contain the preconditioners discussed in [2]. Theoretical analysis shows that all eigenvalues of the preconditioned matrices are tightly clustered. Numerical experiments demonstrate efficiency of the new method and show that preconditioner $\widehat{\mathscr{M}}_{k, \eta, \varepsilon}$ is more efficient than $\widehat{\mathscr{M}}_{k, t}$.

The remainder of the paper is as follows. In Section 2, we establish new blocktriangular preconditioners for the linear systems (1.2) in the general case $k^{2} \in \mathbf{R}^{+}$, and then the corresponding spectral analysis is presented. In Section 3, we provide numerical examples to examine our analysis. Finally, some conclusions are drawn in Section 4.

\section{New Block-Triangular Preconditioners for Any $k^{2}$}

We consider the saddle-point linear system (1.2) arising from the discretized time-harmonic Maxwell equations in mixed form (1.1) and assume that $k^{2}$ is not an eigenvalue and $k^{2} \in \mathbf{R}^{+}$.

Grief and Schötzau [3] provide the block-diagonal Schur complement-free preconditioner $\mathcal{M}_{k}$ as in (1.6). Using the fact that the matrices $A+B^{T} L^{-1} B$ and $A+M$ are spectrally equivalent, the argumentation-free and Schur complement-free preconditioner $\widehat{\mathscr{M}}_{k}$ is defined in (1.7). Spectral analysis shows that the eigenvalues of the preconditioned saddle-point matrices $\mathcal{M}_{k}^{-1} \AA$ and $\widehat{M}_{k}^{-1} \mathcal{A}$ are strongly clustered when $k^{2}$ is small.

Reference [2] provides the block-triangular Schur complement-free preconditioners for the linear system (1.2). In particular, they considered preconditioning matrices for the general case $k^{2} \in \mathbf{R}^{+}$with

$$
\begin{gathered}
\mathcal{M}_{k, t}=\left(\begin{array}{cc}
A-k^{2} M+t B^{T} L^{-1} B & t B^{T} \\
0 & \frac{1-t}{t} L
\end{array}\right), \\
\widehat{\mathfrak{M}}_{k, t}=\left(\begin{array}{cc}
A+\left(t-k^{2}\right) M & t B^{T} \\
0 & \frac{1-t}{t} L
\end{array}\right),
\end{gathered}
$$

where $1 \neq t>k^{2}$. 
For $k^{2}=0$, [15] provides the block-triangular preconditioner for linear system (1.2):

$$
p_{\eta, \varepsilon}=\left(\begin{array}{cc}
A+\eta B^{T} L^{-1} B & (1-\eta \varepsilon) B^{T} \\
0 & \varepsilon L
\end{array}\right)
$$

where $\eta>0$ and $\varepsilon>0$.

Based on the works in $[2,3,15]$, we provide the following new block-triangular Schur complement-free preconditioners $\mathcal{M}_{k, \eta, \varepsilon}$ and $\widehat{\mathcal{M}}_{k, \eta, \varepsilon}$ :

$$
\begin{gathered}
\mathcal{M}_{k, \eta, \varepsilon}=\left(\begin{array}{cc}
A-k^{2} M+\eta B^{T} L^{-1} B & (1-\eta \varepsilon) B^{T} \\
\varepsilon L
\end{array}\right), \\
\widehat{\mathcal{M}}_{k, \eta, \varepsilon}=\left(\begin{array}{cc}
A+\left(\eta-k^{2}\right) M & (1-\eta \varepsilon) B^{T} \\
0 & \varepsilon L
\end{array}\right),
\end{gathered}
$$

where $\eta>k^{2}$ and $\varepsilon \neq 0$ are scaling parameters. It is interesting to note that when parameters $\eta=t$ and $\varepsilon=(1-t) / t, \mathcal{M}_{k, \eta, \varepsilon}$ and $\widehat{\mathscr{M}}_{k, \eta, \varepsilon}$ apparently reduce to $\mathcal{M}_{k, t}$ and $\widehat{\mathscr{M}}_{k, t}$, respectively. We also see that when $k^{2}=0$, the preconditioner $\mathcal{M}_{k, \eta, \varepsilon}$ in (2.3) $(\varepsilon \neq 0)$ is different from $p_{\eta, \varepsilon}$ in (2.2) $(\varepsilon>0)$.

We stress that $\mathcal{M}_{k, \eta, \varepsilon}$ is not the preconditioner we eventually use in actual computation. It is only introduced to lay theoretical basis and motivation for the preconditioner $\widehat{\mathscr{M}}_{k, \eta, \varepsilon}$ in (2.4), which we will use in practice. We note that the $(1,1)$ block $A-k^{2} M+\eta B^{T} L^{-1} B$ in $\mathscr{M}_{k, \eta, \varepsilon}$ is symmetric positive definite for $k$ is sufficiently small [3]. But this is not true when $k$ is large enough. However, this situation may not appear in the actual preconditioner $\widehat{\mathscr{M}}_{k, \eta, \varepsilon}$. The $(1,1)$ block $A+\left(\eta-k^{2}\right) M$ in $\widehat{\mathscr{M}}_{k, \eta, \varepsilon}$ is always symmetric positive definite when $\eta>k^{2}$. In this paper, we will apply the BiCGSTAB with the preconditioner $\widehat{\mathscr{M}}_{k, \eta, \varepsilon}$ as an outer solver for the saddlepoint system (1.2). Then, the overall computational cost of solution procedure relies on how to efficiently solve the linear systems $A+\tau M\left(\tau=\eta-k^{2}\right)$ and $L$, which are called by inner solvers. For the linear system $L$ arising from a standard scalar elliptic problem, many efficient solution methods exist. On the other hand, for solving the linear system $A+\tau M$, we refer to $[6,8,9,14]$, and some detailed numerical examples are provided in [29].

For the spectral analysis, we recall some results which are contained in the following lemma.

Lemma 2.1 (see [3]). The following relations hold:

(i) $\mathcal{R}^{n}=\operatorname{null}(A) \oplus \operatorname{null}(B)$;

(ii) $\left\langle M u_{A}, u_{B}\right\rangle=0$ for any $u_{A} \in \operatorname{null}(A)$ and any $u_{B} \in \operatorname{null}(B)$;

(iii) $\left\langle B^{T} L^{-1} B u_{A}, u_{A}\right\rangle=\left\langle M u_{A}, u_{A}\right\rangle$ for any $u_{A} \in \operatorname{null}(A)$.

Theorem 2.2. Let $\mathcal{A}$ be the saddle-point matrix in (1.2). Then the matrix $\mathfrak{M}_{k, \eta, \varepsilon}^{-1} \mathcal{A}$ has two distinct eigenvalues, which are given by

$$
\lambda_{1}=1, \quad \lambda_{2}=-\frac{1}{\varepsilon\left(\eta-k^{2}\right)}
$$

with the algebraic multiplicities $n$ and $m$, respectively. 
Proof. Suppose that $\lambda$ is an eigenvalue of $\mathcal{M}_{k, \eta, \varepsilon}^{-1} \mathcal{A}$, whose eigenvector is $\left(\begin{array}{l}v \\ q\end{array}\right)$. Then the corresponding eigenvalue problem is

$$
\left(\begin{array}{cc}
A-k^{2} M & B^{T} \\
B & 0
\end{array}\right)\left(\begin{array}{l}
v \\
q
\end{array}\right)=\lambda\left(\begin{array}{cc}
A-k^{2} M+\eta B^{T} L^{-1} B & (1-\eta \varepsilon) B^{T} \\
0 & \varepsilon L
\end{array}\right)\left(\begin{array}{l}
v \\
q
\end{array}\right) .
$$

From the second row we can obtain $q=(1 / \lambda \varepsilon) L^{-1} B v$. By substituting it into the first row we have

$$
(1-\lambda)\left[\lambda\left(A-k^{2} M\right) v+\left(\frac{1}{\varepsilon}+\lambda \eta\right) B^{T} L^{-1} B v\right]=0
$$

It is straightforward to see that any vector $v \in R^{n}$ satisfies (2.7) with $\lambda=1$, so $\lambda=1$ is an eigenvalue of $\mathcal{M}_{k, \eta, \varepsilon}^{-1} \mathcal{A}$. By the similar technique of linear independence considerations from [3], we can demonstrate that the eigenvalue $\lambda=1$ has algebraic multiplicity $n$.

Since there are $m$ linearly independent null vectors of $A$, by Lemma 2.1,

$$
v=v_{A}+v_{B}\left(v_{A} \neq 0\right), \quad v_{A} \in \operatorname{null}(A), v_{B} \in \operatorname{null}(B) .
$$

By Lemma 2.1 (ii) and (iii), and using the inner product in (2.7) with $v_{A}$, we have

$$
(1-\lambda)\left[\frac{1}{\varepsilon}+\lambda\left(\eta-k^{2}\right)\right]\left|v_{A}\right|_{M}^{2}=0 .
$$

Since $v_{A} \neq 0$, from (2.9) we can obtain that $\lambda=-1 / \varepsilon\left(\eta-k^{2}\right)$ is another eigenvalue of $\mathcal{M}_{k, \eta, \varepsilon}^{-1} \mathcal{A}$, and we claim that the eigenvalue $\lambda=-1 / \varepsilon\left(\eta-k^{2}\right)$ has algebraic multiplicity $m$.

Corollary 2.3. Let $-1 / \varepsilon=\eta-k^{2}$. Then the corresponding preconditioned matrix $\mathcal{M}_{k, \eta, \mathcal{E}}^{-1} \mathbb{A}$ has only one eigenvalue $\lambda=1$ of algebraic multiplicity $n+m$.

Proof. From Theorem 2.2, we can easily obtain the corresponding conclusion.

Remark 2.4. From Theorem 2.2, we demonstrate that the preconditioned matrix $\mathcal{M}_{k, \eta, \varepsilon}^{-1} \mathscr{A}$ has precisely two distinct eigenvalues. Then if Krylov subspace methods are used to solve (1.2) with $\mathcal{M}_{k, \eta, \varepsilon}$ as a preconditioner, the iteration will require merely two steps if round-off errors are ignored [30]. And from Corollary 2.3, for any $\eta$, we can find a number $\varepsilon$ which makes the preconditioned matrix $\mathcal{M}_{k, \eta, \varepsilon}^{-1} \mathcal{A}$ have only one eigenvalue. Therefore, we can demonstrate that our preconditioners are more efficient than the block-triangular preconditioner proposed in [2].

Remark 2.5. From (2.3) we know that if $\eta \varepsilon=1$, the new preconditioner reduces to the diagonal preconditioner $\boldsymbol{M}_{k, \eta}$ :

$$
\mathcal{M}_{k, \eta}=\left(\begin{array}{cc}
A-k^{2} M+\eta B^{T} L^{-1} B & 0 \\
0 & \frac{1}{\eta} L
\end{array}\right) .
$$

Then we can use MINRES to solve the linear system (1.2). 
Theorem 2.6. Let $A$ be the saddle-point matrix in (1.2). Then

$$
\lambda_{1}=1, \quad \lambda_{2}=-\frac{1}{\varepsilon\left(\eta-k^{2}\right)}
$$

are the eigenvalues of $\widehat{\mathbb{M}}_{k, \eta, \varepsilon}^{-1} \mathscr{A}$, having algebraic multiplicity $m$. The rest of the eigenvalues satisfy

$$
\frac{\bar{\alpha}-k^{2}}{\bar{\alpha}+\eta-k^{2}} \leq \lambda<1
$$

where $\bar{\alpha}$ is defined as in (1.4).

Proof. Suppose that $\lambda$ is an eigenvalue of $\widehat{M}_{k, \eta, \varepsilon}^{-1} \mathcal{A}$, whose eigenvector is $\left(\begin{array}{l}v \\ q\end{array}\right)$. Then the corresponding eigenvalue problem is

$$
\left(\begin{array}{cc}
A-k^{2} M & B^{T} \\
B & 0
\end{array}\right)\left(\begin{array}{l}
v \\
q
\end{array}\right)=\lambda\left(\begin{array}{cc}
A+\left(\eta-k^{2}\right) M & (1-\eta \varepsilon) B^{T} \\
0 & \varepsilon L
\end{array}\right)\left(\begin{array}{l}
v \\
q
\end{array}\right) .
$$

From the second row we can obtain $q=(1 / \varepsilon \mathcal{\varepsilon})\left(L^{-1} B v\right)$. By substituting it into the first row we have

$$
\lambda\left(A-k^{2} M\right) v+\frac{1}{\varepsilon} B^{T} L^{-1} B v=\lambda^{2}\left(A+\left(\eta-k^{2}\right) M\right) v+\frac{\lambda}{\varepsilon}(1-\eta \varepsilon) B^{T} L^{-1} B v .
$$

Consider the $m$ linearly independent null vectors of $A$, by Lemma 2.1 (i),

$$
v=v_{A}+v_{B} \quad\left(v_{A} \neq 0\right)
$$

where $v_{A} \in \operatorname{null}(A)$ and $v_{B} \in \operatorname{null}(B)$. By Lemma 2.1 (ii) and (iii), and taking the inner product in (2.14) with $v_{A}$, we obtain

$$
(1-\lambda)\left[\frac{1}{\varepsilon}+\lambda\left(\eta-k^{2}\right)\right]\left|v_{A}\right|_{M}^{2}=0
$$

Since $\left|v_{A}\right|_{M} \neq 0, \lambda_{1}=1$ and $\lambda_{2}=-1 /\left(\varepsilon\left(\eta-k^{2}\right)\right)$ are two eigenvalues of $\widehat{\mathcal{M}}_{k, \eta, \varepsilon}^{-1} \mathcal{A}$ and by the similar technique of linear independence considerations from [3], we claim that each eigenvalue has algebraic multiplicity $m$.

For the rest of eigenvectors we have $v_{B} \neq 0$. Noting that

$$
\left\langle B^{T} L^{-1} B v_{A}, v_{B}\right\rangle=\left\langle L^{-1} B v_{A}, B v_{B}\right\rangle=0,
$$

by Lemma 2.1 (ii) and by taking the inner product in (2.14) with $v_{B}$ and using (2.17), we obtain

$$
(1-\lambda)\left|v_{B}\right|_{A}^{2}=\left(\lambda\left(\eta-k^{2}\right)+k^{2}\right)\left|v_{B}\right|_{M}^{2}
$$


It is impossible to have $\lambda=1$, since (2.18) leads to $\left|v_{B}\right|_{M}=0$, which contradicts with $v_{B} \neq 0$. We cannot have $\lambda>1$, since the left-hand side is negative but the right-hand side is positive (because we assume $\eta>k^{2}$ ). Thus, we claim that $\lambda<1$.

From (1.4), we recall that for any $u \in \operatorname{null}(B),|u|_{A}^{2} \geq \bar{\alpha}|u|_{M}^{2}$ with $\bar{\alpha}=\alpha /(1-\alpha)$. Applying this to (2.18), we have $\left(\lambda\left(\eta-k^{2}\right)+k^{2}\right) /(1-\lambda) \geq \bar{\alpha}$. Since $\eta>k^{2}>0$, we have $\bar{\alpha}+\eta-k^{2}>0$ and

$$
\frac{\bar{\alpha}-k^{2}}{\bar{\alpha}+\eta-k^{2}} \leq \ell<1
$$

Corollary 2.7. Let $-1 / \varepsilon=\eta-k^{2}$. Then the corresponding preconditioned matrix $\widehat{\mathscr{M}}_{k, \eta, \varepsilon} A$ has only one eigenvalue $\lambda=1$ with algebraic multiplicity $2 m$. The remaining eigenvalues satisfy (2.12).

Remark 2.8. From (2.4) we know that when $\eta \varepsilon=1$, the new preconditioner reduces to the diagonal preconditioner $\widehat{\mathbb{M}}_{k, \eta}$ :

$$
\widehat{\widetilde{M}}_{k, \eta}=\left(\begin{array}{cc}
A+\left(\eta-k^{2}\right) M & 0 \\
0 & \frac{1}{\eta} L
\end{array}\right) .
$$

Then we can use MINRES to solve the linear system (1.2).

Remark 2.9. From the proof of Theorem 2.6, we easily see that the new preconditioner $\widehat{\mathscr{M}}_{k, \eta, \varepsilon}$ is also efficient for $k^{2}=0$. Then from (2.12), we conclude that if $-1 / \varepsilon=\eta-k^{2}$ and $k^{2}=0$, then the closer $\eta$ is to 0 and the closer $\left(\bar{\alpha}-k^{2}\right) /\left(\bar{\alpha}+\eta-k^{2}\right)=\bar{\alpha} /(\bar{\alpha}+\eta)$ is to 1 ; that is, the preconditioned matrix has more tightly clustered eigenvalues. For a general case of $k^{2} \in \mathbb{R}^{+}$, we can only obtain similar results when $\bar{\alpha}>k^{2}$. The following numerical experiments show that the closer $\eta$ is to $k^{2}$, the less iteration counts we have used for a fixed $k^{2} \in \mathbb{R}^{+}$. However, choosing $\eta-k^{2}$ too small may result in too large $\varepsilon$, then result in ill-conditioning of $\widehat{\mathscr{M}}_{k, \eta, \varepsilon}$. So we choose $\eta-k^{2}$ to be moderate size in practice.

\section{Numerical Experiments}

The test problem is a two-dimensional time-harmonic Maxwell equations in mixed form (1.1) in a square domain $\Omega=(0<x<1 ; 0<y<1)$. We set the right-hand side function so that the exact solution is given by

$$
u(x, y)=\left(\begin{array}{l}
u_{1}(x, y) \\
u_{2}(x, y)
\end{array}\right)=\left(\begin{array}{c}
y(1-y) \\
x(1-x)
\end{array}\right)
$$

and $p \equiv 0$.

We consider five uniformly refined meshes, which are constructed by subsequently splitting each triangle into four triangles by joining the midpoints of the edges of the triangle. Two of five mesh grids are depicted in Figures 1 and 2. The lowest order elements are used to discretize equations. The matrix sizes on different meshes are given in Table 1. 
Table 1: Values of matrix size of the linear system for five meshes.

\begin{tabular}{lccc}
\hline Mesh & $n$ & $m$ & $n+m$ \\
\hline $8 \times 8$ & 176 & 49 & 225 \\
$16 \times 16$ & 736 & 225 & 961 \\
$32 \times 32$ & 3008 & 961 & 3969 \\
$64 \times 64$ & 12160 & 3969 & 16129 \\
$128 \times 128$ & 48896 & 16129 & 65025 \\
\hline
\end{tabular}

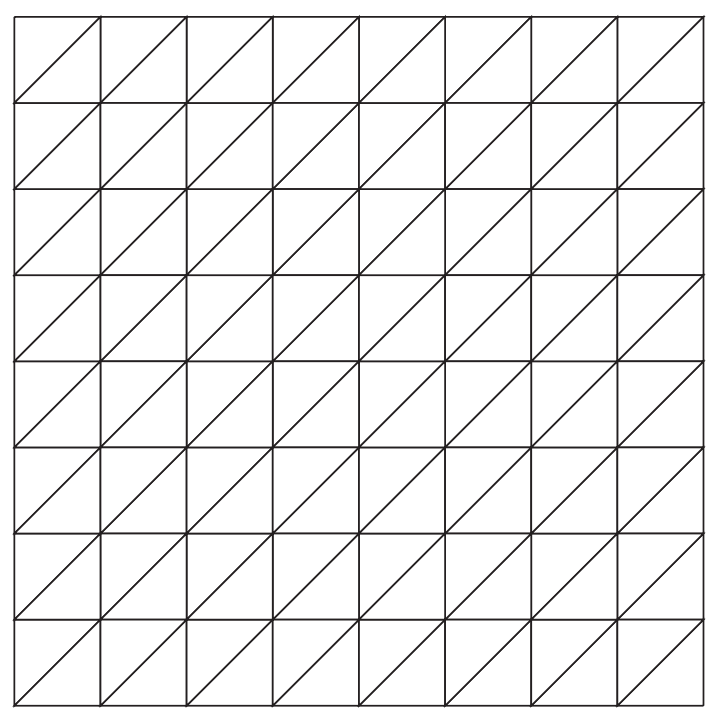

Figure 1: $8 \times 8$ mesh.

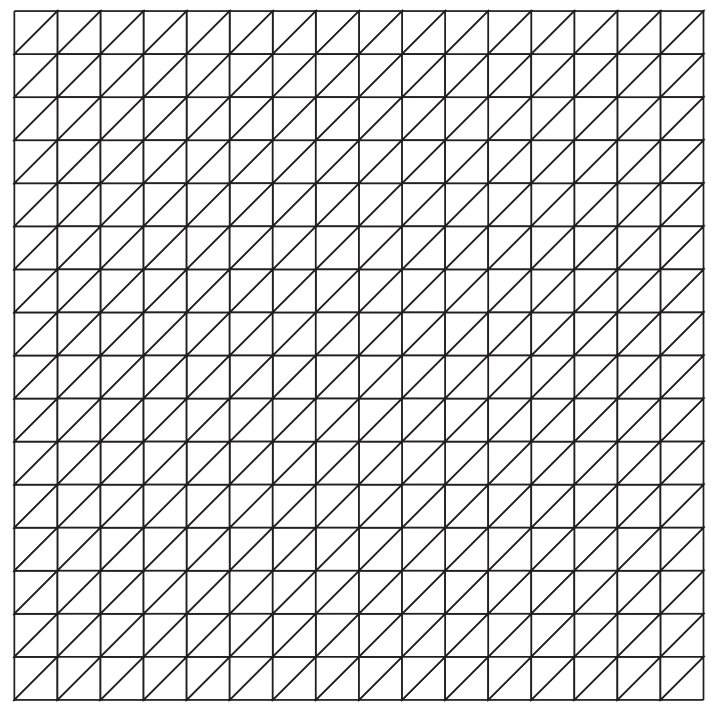

Figure 2: $16 \times 16$ mesh. 


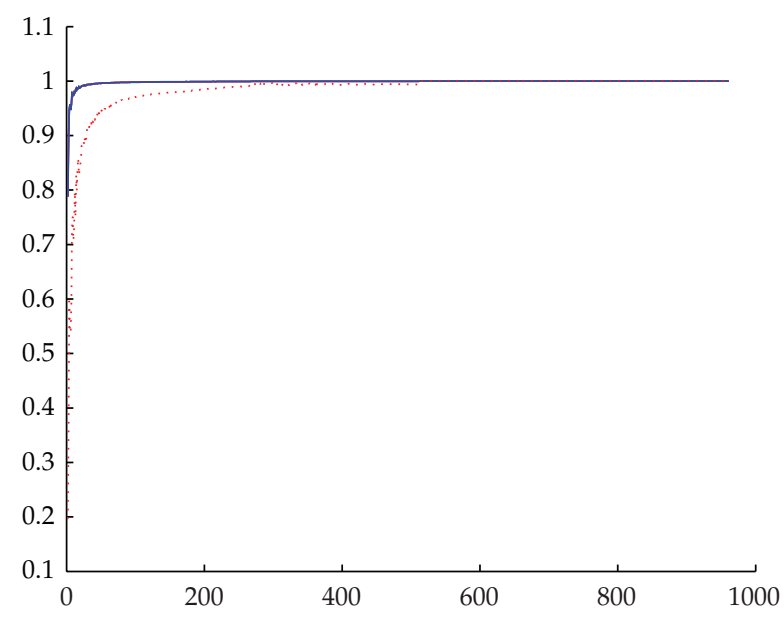

Figure 3: The eigenvalue distribution of the preconditioned matrix $\widehat{M}_{k, \eta, \varepsilon}^{-1}$ A with $k^{2}=2, \eta=2.1$ and $\eta=32$, respectively, and $m+n=961$. The case $\eta=2.1$ is indicated by the solid line while the case $\eta=32$ is indicated by the dotted line.

Our numerical experiments were performed using MATLAB. The machine is a PC-Intel (R), Pentium(R)Dual CPU E2200 2.20 GHz, 1.00 G of RAM. The purpose of our experiments is to investigate the convergence behavior of preconditioned BiCGSTAB by choosing different parameters $\eta$ and and $\varepsilon$ in the preconditioner $\widehat{\mathscr{M}}_{k, \eta, \varepsilon}$. Thus, we apply exact inner solver, and the outer iteration is used as a zero initial guess and stopped when $\left\|r^{(k)}\left(=b-\mathcal{A} x^{(k)}\right)\right\|_{2} /\left\|r^{(0)}(=b)\right\|_{2} \leq 5 \times 10^{-10}$.

From Theorem 2.6 and Corollary 2.7 we know that the preconditioned matrix $\widehat{\mathcal{M}}_{k, \eta, \varepsilon}^{-1} \mathcal{A}$ has one eigenvalue $\lambda=1$, and the remaining eigenvalues are satisfying (2.12). Figure 3 depicts the eigenvalues of the preconditioned matrix $\widehat{\mathbb{M}}_{k, \eta, \varepsilon}^{-1} \mathcal{A}$ with $k^{2}=2$, where $\eta$ and $\varepsilon$ satisfy $-1 / \varepsilon=\eta-k^{2}$, and $m+n=961$. From it we observe that the eigenvalue distribution of preconditioned matrix $\widehat{\mathbb{M}}_{k, \eta, \varepsilon}^{-1} \AA$ with $\eta=k^{2}+0.1=2.1$ denoted by solid line is more tightly clustered than with $\eta=k^{2}+30=32$ denoted by dotted line. From Remark 2.9 we know that the new preconditioner $\widehat{M}_{k, \eta, \varepsilon}$ is also efficient for $k^{2}=0$. Figures $4,5,6$, and 7 show the eigenvalue distribution of the preconditioned matrix $\widehat{\mathbb{M}}_{k, \eta, \varepsilon}^{-1} A$ for different $\eta$ with $k^{2}=0$, where $\eta$ and $\varepsilon$ satisfy $-1 / \varepsilon=\eta-k^{2}$, and $m+n=961$. From Figures $4-7$ we know that the closer the parameter $\eta$ is to 0 , the more tightly clustered the eigenvalues of the preconditioned matrix will be.

Table 2 shows the outer iteration counts for different $k^{2}$ and $\eta$, applying BiCGSTAB with the block-triangular preconditioner, where $\eta$ and $\varepsilon$ satisfy $-1 / \varepsilon=\eta-k^{2}$, and $m+n=$ 16129. The iteration counts are denoted by Iter. We observe that for a fixed $k^{2}$, the closer $\eta$ is to $k^{2}$, the less iteration counts are produced. For comparison, we also give the outer iteration counts for $\eta=t^{*}=1+\left(k^{2}+\sqrt{1+k^{4}}\right) / 2$. We refer the definition of $t^{*}$ to [2]. It shows that the preconditioner $\widehat{\mathscr{M}}_{k, \eta, \varepsilon}$ is more efficient than $\widehat{\mathscr{M}}_{k, t}$.

Tables 3 and 4 show the outer iteration numbers for different meshes, applying BiCGSTAB with the preconditioner $\widehat{\mathscr{M}}_{k, \eta, \varepsilon}$, where $\eta$ are set to be $\eta-k^{2}=0.1$ and $\eta-k^{2}=6$, and $-1 / \varepsilon=\eta-k^{2}$. We observe that the outer iteration numbers of the preconditioned BiCGSTAB are hardly sensitive to the changes in the mesh size. 
Table 2: Iteration counts for different $k^{2}$ and $\eta$, using BiCGSTAB with the preconditioner $\widehat{M}_{k, \eta, \varepsilon}$, and $m+n=$ 16129. The iteration was stopped once $\left\|r^{(k)}\right\| /\left\|r^{(0)}\right\| \leq 5 \times 10^{-10}$.

\begin{tabular}{lccccccccc}
\hline$\eta$ & $k^{2}$ & 0.1 & 0.2 & 0.5 & 1 & 2 & 3 & 4 & 5 \\
\hline$k^{2}+0.001$ & Iter & 2 & 2 & 2.5 & 2.5 & 3 & 3.5 & 4 & 4.5 \\
$k^{2}+0.01$ & Iter & 2 & 2 & 2.5 & 2.5 & 3 & 3.5 & 4 & 4.5 \\
$k^{2}+0.1$ & Iter & 2 & 2 & 2.5 & 2.5 & 3 & 3.5 & 4 & 4.5 \\
$k^{2}+0.25$ & Iter & 2.5 & 2.5 & 2.5 & 2.5 & 3.5 & 3.5 & 4 & 4.5 \\
$k^{2}+0.5$ & Iter & 2.5 & 2.5 & 2.5 & 2.5 & 3.5 & 4 & 4 & 4.5 \\
$k^{2}+1$ & Iter & 2.5 & 2.5 & 2.5 & 3 & 3.5 & 4 & 4 & 4.5 \\
$k^{2}+1.5$ & Iter & 2.5 & 2.5 & 3 & 3 & 3.5 & 4 & 4.5 & 4.5 \\
$k^{2}+2$ & Iter & 3 & 3 & 3 & 3.5 & 3.5 & 4 & 4.5 & 4.5 \\
$k^{2}+5$ & Iter & 3.5 & 3.5 & 3.5 & 4 & 4.5 & 4.5 & 4.5 & 5 \\
$k^{2}+15$ & Iter & 4.5 & 5 & 5 & 5 & 5 & 5.5 & 5.5 & 5.5 \\
$k^{2}+30$ & Iter & 5.5 & 5.5 & 5.5 & 6 & 6 & 6 & 6 & 6 \\
$t^{*}=1+\left(\left(k^{2}+\sqrt{1+k^{4}}\right) / 2\right)$ & Iter & 3 & 3 & 3 & 3 & 3.5 & 4 & 4 & 4.5 \\
\hline
\end{tabular}

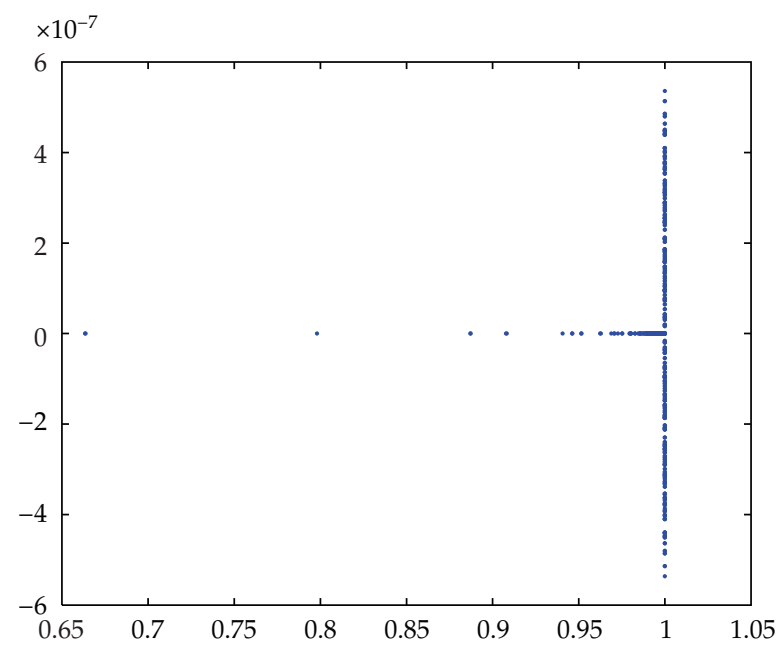

Figure 4: The eigenvalue distribution of the preconditioned matrix $\widehat{M}_{k, \eta, \varepsilon}^{-1} \mathscr{A}$ with $k^{2}=0, \eta=5$ and $m+n=$ 961.

\section{Conclusions}

We have investigated the use of new block-triangular preconditioners with two variable relaxation parameters for solving the mixed formulation of the time-harmonic Maxwell equations. Our results are extensions of the work in $[2,3,15]$. The preconditioned matrices are demonstrated to have clustering eigenvalues. We have shown experimentally that the outer iteration numbers of BiCGSTAB with the new preconditioner are hardly any sensitive to the changes in the mesh size.

\section{Acknowledgments}

The authors thank the anonymous referees for their valuable comments and suggestions which lead to an improved presentation of this paper. This work is supported by the Chinese 
Table 3: Iteration counts for different meshes, using BiCGSTAB with the preconditioner $\widehat{M}_{k, \eta, \varepsilon}$ satisfying $\eta-k^{2}=0.1$ and $-1 / \varepsilon=\eta-k^{2}$. The iteration was stopped once $\left\|r^{(k)}\right\| /\left\|r^{(0)}\right\| \leq 5 \times 10^{-10}$.

\begin{tabular}{lcccccccc}
\hline Mesh & $k^{2}=0$ & $k^{2}=0.25$ & $k^{2}=0.5$ & $k^{2}=1$ & $k^{2}=3$ & $k^{2}=4$ & $k^{2}=6$ & $k^{2}=10$ \\
\hline $8 \times 8$ & 2 & 2.5 & 2.5 & 2.5 & 3.5 & 4 & 4.5 & 5.5 \\
$16 \times 16$ & 2 & 2.5 & 2.5 & 2.5 & 3.5 & 4 & 4.5 & 5 \\
$32 \times 32$ & 2 & 2.5 & 2.5 & 2.5 & 3.5 & 4 & 4.5 & 5.5 \\
$64 \times 64$ & 2 & 2.5 & 2.5 & 2.5 & 3.5 & 4 & 5 & 5.5 \\
$128 \times 128$ & 2 & 2.5 & 3 & 3 & 4 & 4.5 & 5 & 6 \\
\hline
\end{tabular}

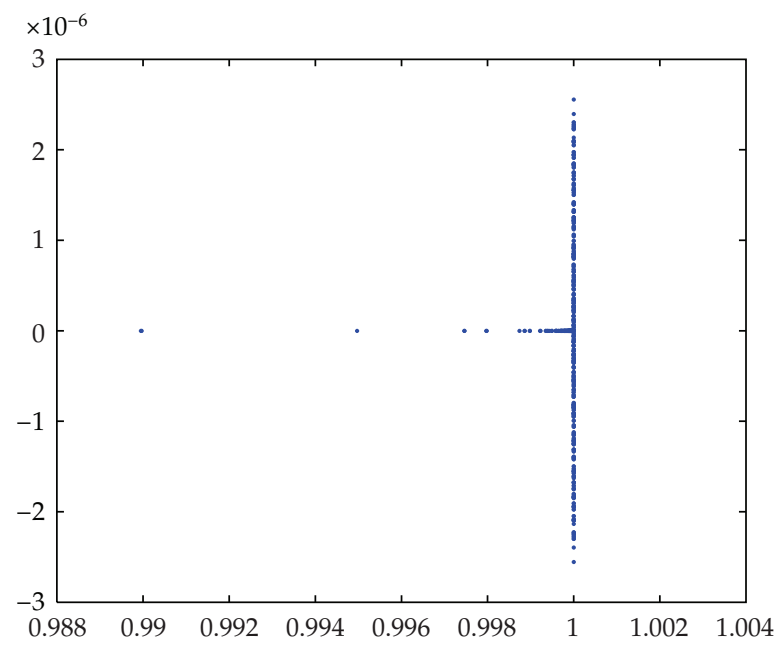

Figure 5: The eigenvalue distribution of the preconditioned matrix $\widehat{\mathscr{M}}_{k, \eta, \varepsilon}^{-1} \mathbb{A}$ with $k^{2}=0, \eta=0.1$ and $m+n=961$.

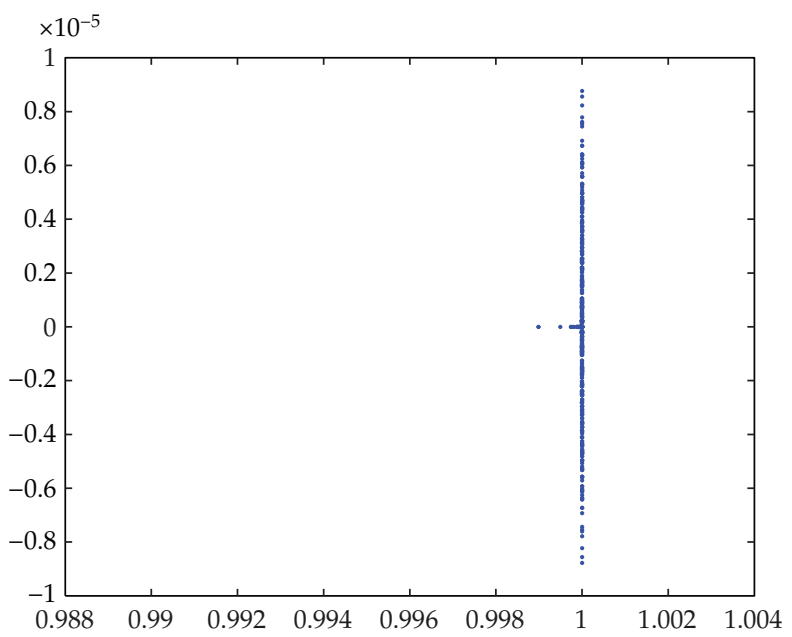

Figure 6: The eigenvalue distribution of the preconditioned matrix $\widehat{\mathbb{M}}_{k, \eta, \varepsilon}^{-1} \mathcal{A}$ with $k^{2}=0, \eta=0.01$ and $m+n=961$. 
Table 4: Iteration counts for different meshes, using BiCGSTAB with the preconditioner $\widehat{M}_{k, \eta, \varepsilon}$ satisfying $\eta-k^{2}=6$ and $-1 / \varepsilon=\eta-k^{2}$. The iteration was stopped once $\left\|r^{(k)}\right\| /\left\|r^{(0)}\right\| \leq 5 \times 10^{-10}$.

\begin{tabular}{lcccccccc}
\hline Mesh & $k^{2}=0$ & $k^{2}=0.25$ & $k^{2}=0.5$ & $k^{2}=1$ & $k^{2}=3$ & $k^{2}=4$ & $k^{2}=6$ & $k^{2}=10$ \\
\hline $8 \times 8$ & 3.5 & 3.5 & 3.5 & 4 & 4.5 & 4.5 & 5 & 6.5 \\
$16 \times 16$ & 3.5 & 4 & 4 & 4 & 4.5 & 4.5 & 5.5 & 6.5 \\
$32 \times 32$ & 3.5 & 4 & 4 & 4 & 4.5 & 4.5 & 5.5 & 6 \\
$64 \times 64$ & 3.5 & 4 & 4 & 4 & 4.5 & 5 & 5.5 & 6 \\
$128 \times 128$ & 3.5 & 4 & 4.5 & 4.5 & 5 & 5 & 6 & 6.5 \\
\hline
\end{tabular}

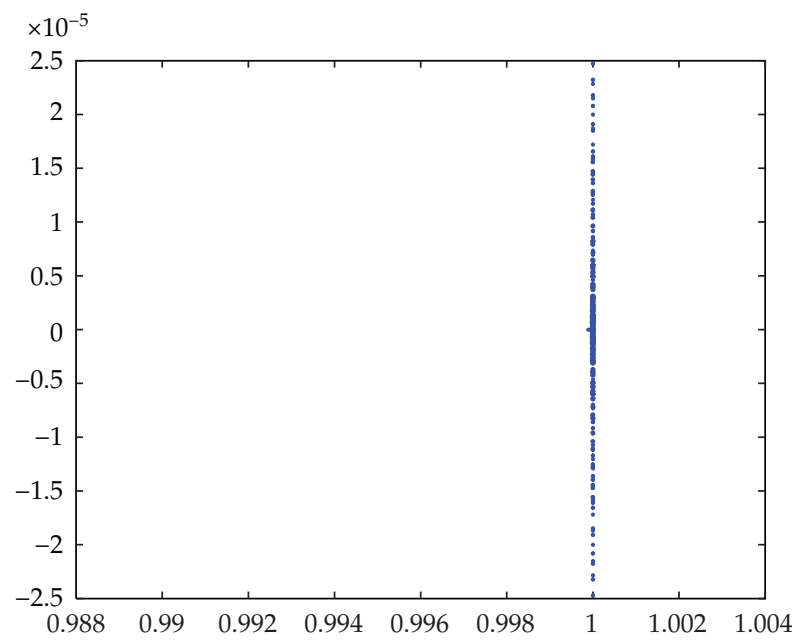

Figure 7: The eigenvalue distribution of the preconditioned matrix $\widehat{\mathscr{M}}_{k, \eta, \varepsilon}^{-1} A$ with $k^{2}=0, \eta=0.001$ and $m+n=961$.

National Science Foundation Project (11161014), the Science and Technology Development Foundation of Guangxi (Grant no. 0731018), and Innovation Project of Guangxi Graduate Education (Grant no. ZYC0430).

\section{References}

[1] Z. Chen, Q. Du, and J. Zou, "Finite element methods with matching and nonmatching meshes for Maxwell equations with discontinuous coefficients," SIAM Journal on Numerical Analysis, vol. 37, no. 5, pp. 1542-1570, 2000.

[2] G.-H. Cheng, T.-Z. Huang, and S.-Q. Shen, "Block triangular preconditioners for the discretized timeharmonic Maxwell equations in mixed form," Computer Physics Communications, vol. 180, no. 2, pp. 192-196, 2009.

[3] C. Greif and D. Schötzau, "Preconditioners for the discretized time-harmonic Maxwell equations in mixed form," Numerical Linear Algebra with Applications, vol. 14, no. 4, pp. 281-297, 2007.

[4] Q.-Y. Hu and J. Zou, "Substructuring preconditioners for saddle-point problems arising from Maxwell's equations in three dimensions," Mathematics of Computation, vol. 73, no. 245, pp. 35-61, 2004.

[5] I. Perugia, D. Schötzau, and P. Monk, "Stabilized interior penalty methods for the time-harmonic Maxwell equations," Computer Methods in Applied Mechanics and Engineering, vol. 191, no. 41-42, pp. 4675-4697, 2002.

[6] D. N. Arnold, R. S. Falk, and R. Winther, "Multigrid in H(div) and H(curl)," Numerische Mathematik, vol. 85, no. 2, pp. 197-217, 2000.

[7] J. Gopalakrishnan, J. E. Pasciak, and L. F. Demkowicz, "Analysis of a multigrid algorithm for time harmonic Maxwell equations," SIAM Journal on Numerical Analysis, vol. 42, no. 1, pp. 90-108, 2004. 
[8] R. Hiptmair, "Multigrid method for Maxwell's equations," SIAM Journal on Numerical Analysis, vol. 36, no. 1, pp. 204-225, 1999.

[9] S. Reitzinger and J. Schöberl, "An algebraic multigrid method for finite element discretizations with edge elements," Numerical Linear Algebra with Applications, vol. 9, no. 3, pp. 223-238, 2002.

[10] A. Alonso and A. Valli, "An optimal domain decomposition preconditioner for low-frequency timeharmonic Maxwell equations," Mathematics of Computation, vol. 68, no. 226, pp. 607-631, 1999.

[11] Q.-Y. Hu and J. Zou, "A nonoverlapping domain decomposition method for Maxwell's equations in three dimensions," SIAM Journal on Numerical Analysis, vol. 41, no. 5, pp. 1682-1708, 2003.

[12] A. Toselli, “Overlapping Schwarz methods for Maxwell's equations in three dimensions," Numerische Mathematik, vol. 86, no. 4, pp. 733-752, 2000.

[13] A. Toselli, O. B. Widlund, and B. I. Wohlmuth, "An iterative substructuring method for Maxwell's equations in two dimensions," Mathematics of Computation, vol. 70, no. 235, pp. 935-949, 2000.

[14] R. Hiptmair and J. Xu, "Nodal auxiliary space preconditioning in H(curl) and H(div) spaces," SIAM Journal on Numerical Analysis, vol. 45, no. 6, pp. 2483-2509, 2007.

[15] T.-Z. Huang, L.-T. Zhang, T.-X. Gu, and X.-Y. Zuo, "New block triangular preconditioner for linear systems arising from the discretized time-harmonic Maxwell equations," Computer Physics Communications, vol. 180, no. 10, pp. 1853-1859, 2009.

[16] Z.-Z. Bai, B. N. Parlett, and Z.-Q. Wang, "On generalized successive overrelaxation methods for augmented linear systems," Numerische Mathematik, vol. 102, no. 1, pp. 1-38, 2005.

[17] Z.-Z. Bai and Z.-Q. Wang, "On parameterized inexact Uzawa methods for generalized saddle point problems," Linear Algebra and Its Applications, vol. 428, no. 11-12, pp. 2900-2932, 2008.

[18] Z.-Z. Bai, "Structured preconditioners for nonsingular matrices of block two-by-two structures," Mathematics of Computation, vol. 75, no. 254, pp. 791-815, 2006.

[19] Z.-Z. Bai and G. H. Golub, "Accelerated Hermitian and skew-Hermitian splitting iteration methods for saddle-point problems," IMA Journal of Numerical Analysis, vol. 27, no. 1, pp. 1-23, 2007.

[20] Z.-Z. Bai, G. H. Golub, and C.-K. Li, "Convergence properties of preconditioned Hermitian and skew-Hermitian splitting methods for non-Hermitian positive semidefinite matrices," Mathematics of Computation, vol. 76, no. 257, pp. 287-298, 2007.

[21] Z.-Z. Bai, G. H. Golub, and M. K. Ng, "Hermitian and skew-Hermitian splitting methods for nonHermitian positive definite linear systems," SIAM Journal on Matrix Analysis and Applications, vol. 24 no. 3, pp. 603-626-626, 2003.

[22] Z.-Z. Bai, G. H. Golub, and J.-Y. Pan, "Preconditioned Hermitian and skew-Hermitian splitting methods for non-Hermitian positive semidefinite linear systems," Numerische Mathematik, vol. 98, no. 1, pp. 1-32, 2004.

[23] Z.-Z. Bai, M. K. Ng, and Z.-Q. Wang, "Constraint preconditioners for symmetric indefinite matrices," SIAM Journal on Matrix Analysis and Applications, vol. 31, no. 2, pp. 410-433, 2009.

[24] Z.-Z. Bai and Z.-Q. Wang, "Restrictive preconditioners for conjugate gradient methods for symmetric positive definite linear systems," Journal of Computational and Applied Mathematics, vol. 187, no. 2, pp. 202-226, 2006.

[25] P. B. Monk, "A mixed method for approximating Maxwell's equations," SIAM Journal on Numerical Analysis, vol. 28, no. 6, pp. 1610-1634, 1991.

[26] P. Monk, Finite Elements For Maxwell's Equations, Oxford University Press, New York, NY, USA, 2003.

[27] J.-C. Nédélec, "Mixed finite elements in $\mathbb{R}^{3}$," Numerische Mathematik, vol. 35, no. 3, pp. 315-341, 1980.

[28] C. Grief and D. Schötzau, "Preconditioers for saddle point linear systems with highly singular $(1,1)$ blocks, ENTA," Special Volume on Saddle Point Systems, vol. 22, pp. 114-121, 2006.

[29] D. Li, C. Grief, and D. Schotzau, "Parallel numerical solution of the timeharmonic Maxwell equations in mixed form," Linear Algebra and Its Applications, vol. 19, no. 3, pp. 525-539, 2012.

[30] A. Greenbaum, Iterative Methods for Solving Linear Ystems, vol. 17, SIAM, Philadelphia, Pa, USA, 1997. 


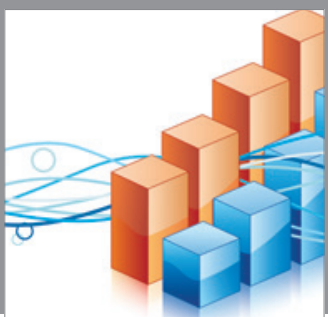

Advances in

Operations Research

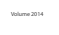

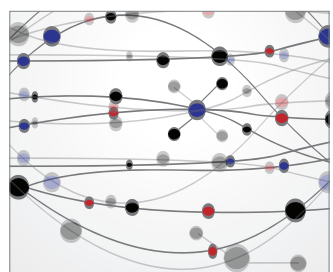

\section{The Scientific} World Journal
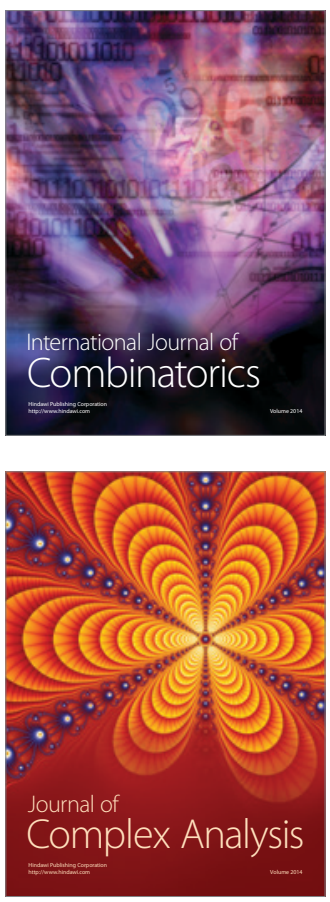

International Journal of

Mathematics and

Mathematical

Sciences
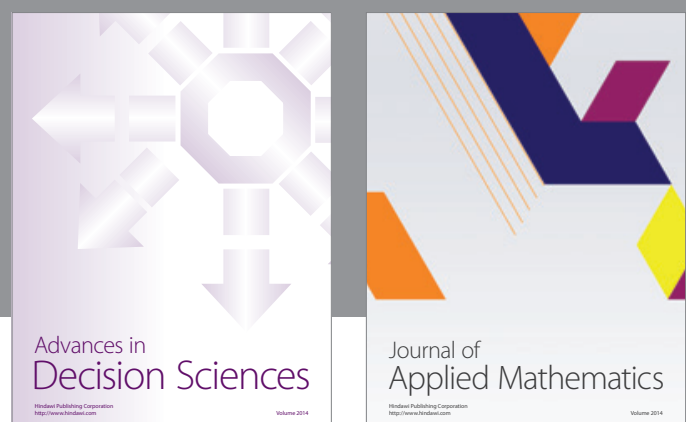

Journal of

Applied Mathematics
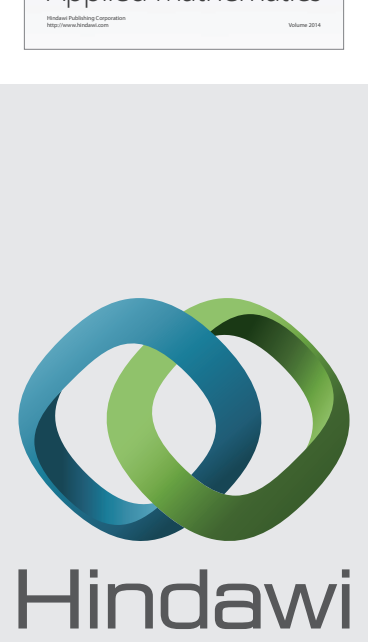

Submit your manuscripts at http://www.hindawi.com
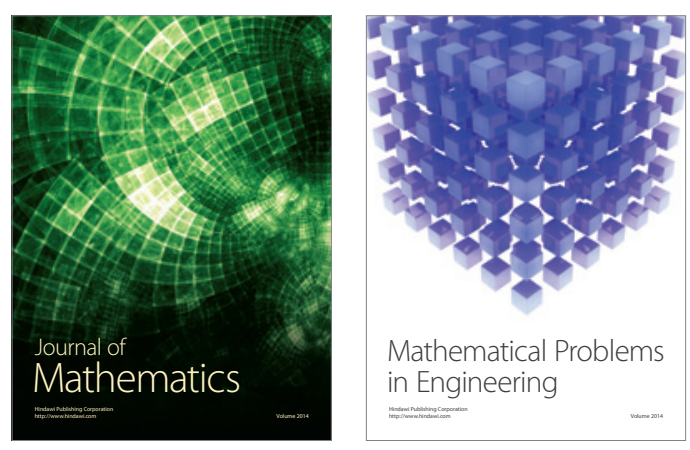

Mathematical Problems in Engineering
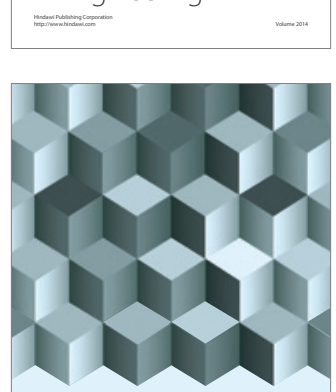

Journal of

Function Spaces
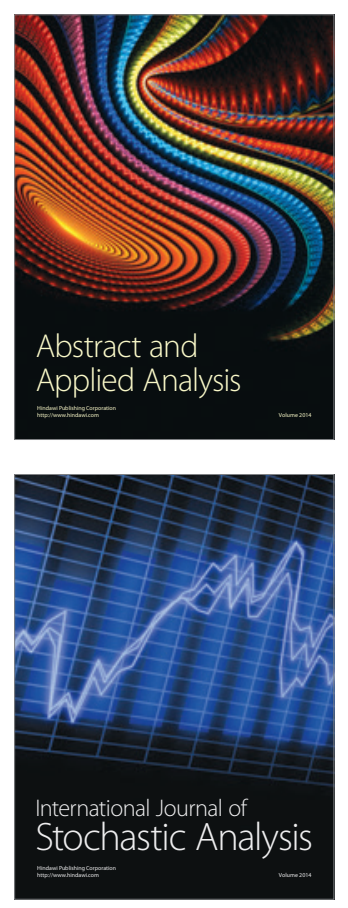

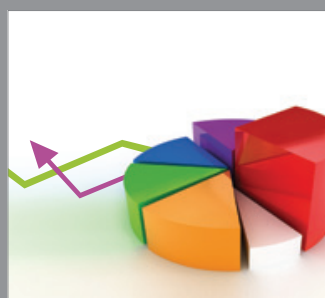

ournal of

Probability and Statistics

Promensencen
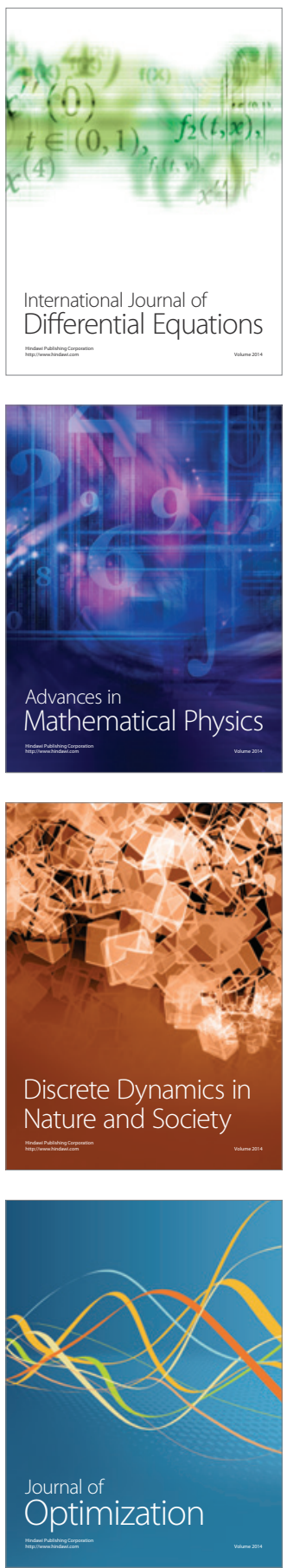